\title{
How Rich is Platelet Rich Plasma? A Simple Preparation Method of PRP and Its Quality According to Various Classification Systems
}

\author{
Felice FAIZAL ${ }^{1}$, Balachandra NAMBIAR², Raja CHATTERJEE', Praveer RANJAN
}

\begin{abstract}
Background: platelets are a rich source of various growth factors and hence platelet rich plasma (PRP) is being used therapeutically in the field of dermatology, orthopaedics and dentistry with promising results. However, methods of preparation of PRP vary with many commercial kits also available. Scoring systems like DEPA score, PAW system and sports medicine criteria score help in determining the quality of the final product and guide therapy. Here we test a simple two step method for preparation of PRP and score it according to the abovementioned criteria while comparing it with the commercial kits analysed in literature. Methods: $10 \mathrm{ml}$ whole blood was collected in $1.5 \mathrm{ml}$ ACD solution from 100 healthy willing participants and analysed for platelet concentration. Sample was then centrifuged at $1600 \mathrm{rpm} \times 4$ minutes in a calibrated laboratory centrifuge and supernatant was collected in a sterile tube using a sterile syringe. The supernatant was further concentrated for platelets by centrifuging at $3600 \mathrm{rpm} X$ 10 minutes to form a platelet pellet at the bottom. All but $1 \mathrm{ml}$ supernatant (Platelet deficient plasma) was discarded and the platelet pellet was suspended again to form platelet rich plasma. Platelet count was estimated in this sample as done with the whole blood sample. The results were compared and analysed using statistical methods to determine the efficacy of concentrating platelets by this method. Results: The tested method gave a final concentration of more than four times the baseline concentration with approximately $30 \%$ efficiency of capture and $80 \%$ purity. This was comparable to majority of the commercial systems tested in literature at a fraction of the cost. The scores according to various classification systems were determined and reported. Conclusion: Every platelet rich plasma prepared for therapeutic purposes should be classified according to any of the available scoring systems to determine its suitability for the purpose and to maintain uniformity in the quality of the product. The proposed two step method of preparation yields satisfactory quality of PRP by utilizing services of a basic laboratory at a fraction of the cost of commercial systems thereby enabling smaller medical institutions to utilize PRP therapy.
\end{abstract}

Keywords: androgenic alopecia, growth factors, platelet rich plasma.

1 Department of Pathology, Army Medical Corps, India

${ }^{2}$ Department of Anaesthesiology, Army Medical Corps, India
Corresponding author.

Felice FAIZAL, A 201, Kailash towers, IGNOU road, 110030, New Delhi, India.

E-mail: flcfzl@gmail.com 


\section{INTRODUCTION}

Platelet rich plasma (PRP) has recently gained a lot of attention due to the fact that platelet granules contain a plethora of growth factors including platelet-derived growth factor, transforming growth factor beta 1 , transforming growth factor beta 2 , insulin-like growth factor, epidermal growth factor, epithelial cell growth factor (ECGF) etc ${ }^{1}$. The use of growth factors derived from platelets for various therapeutic procedures have been studied and tried with varied results ${ }^{2-13}$.

Recently, commercially available kits have sprung up which guarantee a higher yield of platelets from a small amount of blood but incur a high cost of production ${ }^{14}$. To circumvent the cost and still reap the probable benefits of this experimental treatment, many practitioners resort to simply centrifuging small amounts of blood without following specific guidelines and use the supernatant so obtained thereby leading to lower yield of platelets, higher amount of dosage in terms of volume, greater degree of pain and ultimately poor $\mathrm{pa}^{-}$ tient acceptance and results.

As the use of PRP is growing exponentially because of the publicity and the easy availability, there needs to be a proper guideline for harvesting platelets from a small amount of blood and to classify the end product in a universal score to determine its quality before being used for a variety of therapeutic purposes including stimulating hair growth in cases of androgenic alopecia, hastening bone formation in cases of autologous bone grafting etc.

Studies have proposed different characterisation criteria for PRP like the DEPA classification which takes the following parameters into account: (1) Dose of injected platelets, (2) Efficiency of production, (3) Purity of PRP and (4) Activation process (Table 1). Other classification methods including PAW system of classification (Figure 1) and the sports medicine criteria take in to account (1) WBC concentration, (2) activation and (3) final concentration (Table 2) ${ }^{15,16}$.

\section{THE PARAMETERS OF PRP}

The different parameters used in these classification systems need to be understood to correctly choose a platelet rich plasma preparation for each specific intended purpose.

A. Platelet concentration/ Dose of injected platelets: the quantification of platelets in PRP can be done either in terms of times increase of baseline count or as an absolute platelet count per microlitre. The best results have been found with platelet concentrations ranging from 2 times to 6 times the baseline platelet concentration corresponding with absolute values greater than baseline and going up to $1800000 / \mathrm{uL}^{17-19}$. The

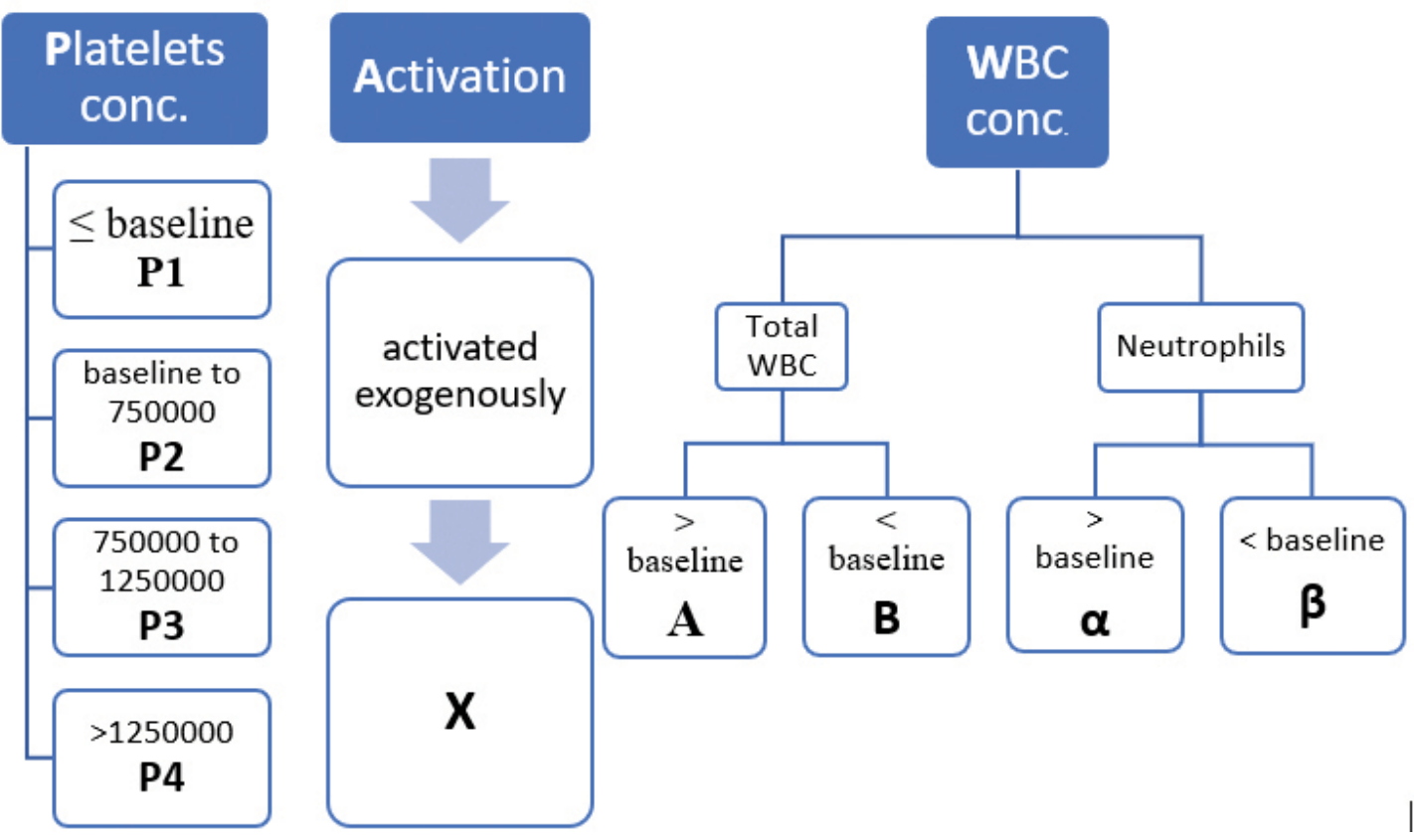

Figure 1. PAW (Platelets, Activation, WBC) SYSTEM OF CLASSIFICATION OF PRP. 


\begin{tabular}{|l|l|l|l|}
\hline $\begin{array}{l}\text { Dosage of platelets injected (in } \\
\text { billions) }\end{array}$ & $\begin{array}{l}\text { Efficiency of preparation } \\
\text { (Platelet recovery rate in \%) }\end{array}$ & $\begin{array}{l}\text { Purity of preparation (\% of platelets in final } \\
\text { PRP) }\end{array}$ & Activation \\
\hline A: $>5:$ Very high dose & A: $>90:$ High & A: $>90:$ Very pure PRP & A: Activated \\
B: 3 to 5: High dose & B: $70-90:$ Medium & B: 70-90: Pure PRP \\
C: 1 to 3: Medium dose & C: $30-70:$ Low & C: $30-70:$ Heterogeneous PRP \\
D: $<1:$ Low dose & D: $<30:$ Poor & D: Whole blood PRP & \\
\hline
\end{tabular}

\section{TABLE 2. SPORTS MEDICINE CRIERIA FOR CLASSIFICATION OF PRP}

\begin{tabular}{|l|l|l|l|}
\hline & WBC & Activation & Conc. of platelets (compared to baseline) \\
\hline Type 1 & Increased & Activated & A:5x or greater B: less than $5 x$ \\
\hline Type 2 & Increased & Not Activated & A:5x or greater B: less than $5 x$ \\
\hline Type 3 & Minimal or no WBC & Activated & A:5x or greater B: less than $5 x$ \\
\hline Type 4 & Minimal or no WBC & Not Activated & A:5x or greater B: less than 5x \\
\hline
\end{tabular}

dose of injected platelets can be determined by multiplying the final volume used by the concentration.

However, it should be borne in mind that higher concentrations don't always mean better results as studies have shown inhibitory effect on growth at concentrations having greater than 6-fold increase in the final product. The underlying mechanism may be a paradoxical inhibition due to alteration in the healing milieu by downregulation of growth factor receptors, receptor desensitisation and stimulation of apoptosis $^{19}$.

B. Efficiency of capture: the efficiency of capture of any method can be calculated by determining the percentage of total platelets from the whole blood sample that have been captured in the PRP. A majority of the commercially available kits analysed by Magalon et al had an efficiency rate ranging from 30 to $70 \% 15$.

C. Purity of preparation/ WBC concentration: $\mathrm{Pu}-$ rity of preparation signifies the relative composition of platelets, leucocytes and RBCs in the obtained PRP with a relative concentration of platelets above $90 \%$ being classified as very pure PRP. The desirability of WBCs in the final product has been under scrutiny and depends on the intended use of the PRP. Therefore, it should be assessed and reported while classifying the end product as either below baseline or above baseline WBC levels.
D. Activation of PRP: Various exogenous methods like calcium chloride, thrombin and type 1 collagen can be added to activate platelets in vitro before use. Whether exogenous activation leads to better results or not needs to be investigated further but adverse effects such as lowering of $\mathrm{pH}$ of $\mathrm{PRP}$ and subsequent increase in pain on addition of calcium chloride need to be considered.

\section{MATERIAL AND METHODS}

Time period: July 2019 to Oct 2019

Study setting: Defence services teaching hospital

Sample size: 100

Inclusion criteria:

a) Samples from consenting healthy individuals who reported for routine medical examination.

b) Samples which did not show presence of platelet clumps on analysis

\section{Exclusion criteria:}

a) Samples from individuals who have reported to the hospital for some illness.

b) Samples which showed presence of platelet clumps on analysis during any step.

\section{Procedure}

a) $10 \mathrm{ml}$ blood was collected in $1.5 \mathrm{ml} \mathrm{ACD}$ solution in sterile tubes and mixed using inversion technique.

b) Whole blood sample was analysed for platelet concentration using Beckman Coulter haematology analyser and results noted. 


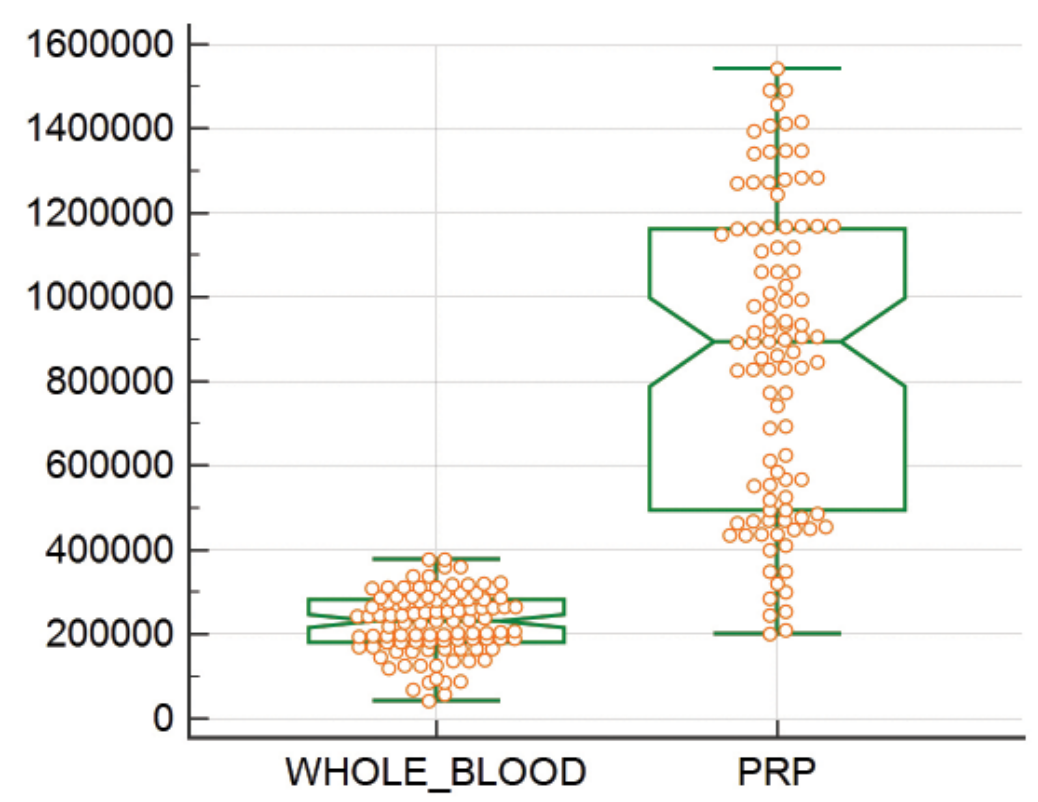

Figure 2. Box and Whisker Plot for Whole Blood and PRP demonstrating the average and the relative increase in platelet concentration in the final product.

c) Sample was then centrifuged at $1600 \mathrm{rpm} \mathrm{X} 4$ minutes in a calibrated laboratory centrifuge and supernatant was collected in a sterile tube using a sterile syringe

d) The supernatant was further concentrated for platelets by centrifuging at $3600 \mathrm{rpm} \mathrm{X} 10 \mathrm{mi}^{-}$ nutes to form a platelet pellet at the bottom. All but $1 \mathrm{ml}$ of the supernatant (Platelet deficient plasma) was discarded and the platelet pellet was resuspended to form $1 \mathrm{ml}$ of platelet rich plasma.

e) Platelet count was estimated in this sample as done with the whole blood sample.

f) The results were compared and analysed using statistical methods to determine the efficacy of concentrating platelets by this method

\section{RESULTS}

PRP prepared from 100 samples by the proposed two step method was evaluated for quality and the following were observed (Figure 2).

Average times increase in platelet concentration: 4.13 times.

Average platelet concentration in the PRP: 853477 platelets / uL.

Average efficiency of capture: $39.8 \%$.

Average purity of PRP: $80.3 \%$.
WBC count: lesser than baseline count in $97 \%$ of samples.

Activation: not activated exogenously.

Therefore, the average final score according to the three different classification systems discussed above would be as follows

1. DEPA Score: if $1 \mathrm{ml}$ of PRP made from $10 \mathrm{ml}$ whole blood: DCB

2. PAW Score: $\mathbf{P} \mathbf{3}-\mathbf{B} \boldsymbol{\beta}$

3. Sports medicine criteria score: Type 4B

\section{DISCUSSION}

The quality of PRP prepared by the abovementioned method was evaluated using the available scoring systems in literature and compared with other studies.

DEPA score: The final DEPA score of our preparation was DCB. Magalon et al did a thorough search of literature and recorded the DEPA scores of PRP obtained from commercial kits and homemade methods used in various studies including their own15.

The dosage of injected platelets was 0.9 billion platelets if $1 \mathrm{ml}$ of PRP was prepared from $10 \mathrm{ml}$ of blood which characterized it as " $D$ " as per Table 1 . Out of the eleven methods which were tested by various researchers and which used similar amount of whole blood to harvest platelets, ten had the same score. Nine other 
methods which used significantly higher amounts of blood generated a higher dosage of platelets which can be matched by the proposed method by increasing the initial volume.

Around $40 \%$ of the platelets from the initial sample were captured in the PRP, thereby giving us an efficiency score of "C". Thirteen of the twenty methods tested in literature fared equally at " $C$ " with 4 having lower ("D") and 2 having better ("B") efficiency. This shows comparable efficiency of the proposed method with commercially available kits.

Purity was determined by relative concentration of platelets among all cellular components of the PRP and ranged from $69 \%$ to $91 \%$ with an average of slightly above $80 \%$. Thus, scored as "B" which was similar to six and better than ten out of the twenty methods analysed by Magalon et al., with only 4 being better in terms of purity 15.

The final score as per other classification systems are P3-B (PAW classification) and type 4B (sports medicine criteria) thereby implying a sufficiently high concentration of platelets with lower count of WBCs

The DEPA system of classification seems to be better while comparing different methods of preparations as it takes into account contamination also by RBCs in addition to $\mathrm{WBC}$ and has a provision for accounting for absolute efficiency of capture. Also, the dosage of platelets can be increased by drawing larger amounts of blood to create higher volume of PRP whereas in the other classification systems the platelet concentrations cannot be improved unless higher amount of blood is used to make same amount of PRP.

The other two systems for classification provide adequate information about the final PRP to guide therapy as per indication. The PRP produced by the proposed method had a score of P3-B $\beta$ in the PAW system and a Sports medicine criteria Type 4B. if exogenously activated the PAW score would be expressed as $\mathrm{P} 3 \mathrm{x} \mathrm{B} \beta$.

\section{CONCLUSION}

Every PRP should be scored according to any of the criteria which suits the purpose of the institution to determine its quality and suitability for the indication. This study illustrates a simple method to harvest a good quality of PRP using the services of a basic laboratory which can prove to be economical and beneficial in terms of patient outcomes in future clinical studies.

The following must be kept in mind while evaluating the final concentration of the platelets

1. The final concentration depends on the baseline platelet count and hydration status of the individual with a higher baseline count and a well hydrated donor yielding a higher final concentration.

2. The centrifugal force applied on the sample may vary between different centrifuges depending on the rotor size of the centrifuge.it is imperative to adjust the other two variables i.e. speed and duration of spin to achieve optimal centrifugal force. The centrifugal force in our method for the specific instrument used was $430 \mathrm{~g}$ x 4 minutes followed by $2200 \mathrm{~g}$ x 10 minutes.

3. Platelet count should not be determined immediately after suspending the platelet pellet as the presence of clumps would give erroneous readings.it is recommended to allow the PRP to be placed on a sample roller for 20 minutes before analysis.

4. The dose of injected platelets may be increased by drawing more blood and following the same procedure to harvest a higher number of platelets.

Compliance with ethics requirements: The authors declare no conflict of interest regarding this article. The authors declare that all the procedures and experiments of this study respect the ethical standards in the Helsinki Declaration of 1975, as revised in 2008(5), as well as the national law. Informed consent was obtained from all the patients included in the study. 
Felice FAIZAL et al.

\section{References}

1. Kiuru J, Viinikka L, Myllyla G, Pesonen K, Perheentupa J. Cytoskeleton-dependent release of human platelet epidermal growth factor. Life Sci 1991;49(26):1997-2003

2. Marx RE, Carlson ER, Eichstaedt RM, Schimmele SR, Strauss $J E$, Georgeff KR. Platelet-rich plasma: Growth factor enhancement for bone grafts. Oral Surg Oral Med Oral Pathol Oral Radiol Endod 1998;85(6):638-646.

3. Anitua E. Plasma rich in growth factors: Preliminary results of use in the preparation of future sites for implants. Int J Oral Maxillofac Implants 1999;14(4):529-535

4. Lynch SE, Buser D, Hernandez RA, et al. Effects of the plateletderived growth factor/insulin-like growth factor-I combination on bone regeneration around titanium dental implants. Results of a pilot study in beagle dogs. J Periodontol 1991;62(11):710716.

5. Becker W, Lynch SE, Lekholm U, et al. A comparison of ePTFE membranes alone or in combination with plateletderived growth factors and insulin-like growth factor-I or demineralized freeze-dried bone in promoting bone formation around immediate extraction socket implants. J Periodontol 1992;63(11):929940.

6. Cho MI, Lin WL, Genco RJ. Platelet-derived growth factor-modulated guided tissue regenerative therapy. J Periodontol 1995; 66(6):522-530.

7. Lynch SE, de Castilla GR, Williams RC, et al. The effects of shortterm application of a combination of plateletderived and insulin-like growth factors on periodontal wound healing. J Periodontol 1991;62(7):458-467.

8. Park JB, Matsuura M, Han KY, et al. Periodontal regeneration in class III furcation defects of beagle dogs using guided tissue regenerative therapy with platelet-derived growth factor. J Periodontol 1995;66(6):462-477.

9. Rutherford RB, Niekrash CE, Kennedy JE, Charette MF. Platelet-derived and insulin-like growth factors stimulate regene- ration of periodontal attachment in monkeys. $\mathrm{J}$ Periodont Res 1992;27(4 Pt 1):285-290.

10. Rutherford RB, Ryan ME, Kennedy JE, Tucker MM, Charette MF. Platelet-derived growth factor and dexamethasone combined with a collagen matrix induce regeneration of the periodontium in monkeys. J Clin Periodontol 1993;20(7):537-544.

11. Schiavone G, Raskovic D, Greco J, Abeni D. Platelet-rich plasma for androgenetic alopecia: A pilot study. Dermatol Surg 2014; 40:1010-9.

12. Betsi EE, Germain E, Kalbermatten DF, Tremp M, Emmenegger V. Platelet-rich plasma injection is effective and safe for the treatment of alopecia. Eur J Plast Surg 2013:36:407-12.

13. Navarro MR, Asín M, Martínez MA, Martinez AM, Molina C, Moscoso $L$, et al. Management of androgenetic alopecia: $A$ comparative clinical study between plasma rich in growth factors and topical minoxidil. Eur J Plast Surg 2016;1:1-8.

14. Correlation of Platelet Concentration in Platelet-rich Plasma to the Extraction Method, Age, Sex, and Platelet Count of the Donor.Gernot Weibrich et al. Int J Oral Maxillofac Implants 2001; 16:693-699.

15. Magalon J, Chateau AL, Bertrand B, et al. DEPA classification: a proposal for standardising PRP use and a retrospective application of available devices. BMJ Open Sport Exerc Med 2016;2:e000060. doi:10.1136/bmjsem-2015000060.

16. Mishra A, Harmon $\mathrm{K}$, Woodall J, et al. Sports medicine applications of platelet rich plasma. Curr Pharm Biotechnol 2012;13: 1185-95

17. Anitua' E, Sanchez M, Zalduendo MM, et al. Fibroblastic response-to -treatment with different preparations rich in growth factors. Cell prolif 2009;42:162-170.

18. Graziani F, Ivanovski C, Cei S, Ducci F, Tonetti M, Gabriele M. The in vitro effect of different PRP concentrations on osteoblasts and fibroblasts. Clin Oral Implants Res 2006;17: 212-219.

19. Weibrich G, Hansen K, Kleis W, Buch R, Hitzler WE. Effect of platelet concentration in platelet-rich plasma on peri-implant bone regeneration. Bone 2004;34:665-671. 